\title{
Análisis del marco normativo de la educación en el contexto global
}

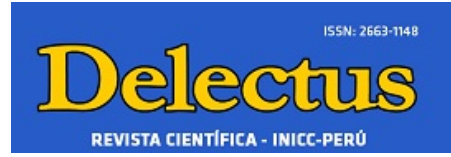

\section{Analysis of the normative framework of education in the global context}

\author{
Villero Pacheco, Manuel Facundo; Tejeira, Yamale
}

\author{
Manuel Facundo Villero Pacheco \\ mfvp03@gmail.com \\ Universidad Metropolitana de Educación Ciencia y \\ Tecnología , Panamá \\ iD Yamale Tejeira \\ yamale.tejeira@up.ac.pa \\ Universidad de Panamá, Panamá
}

\section{Delectus}

Instituto Nacional de Investigación y Capacitación Continua, Perú ISSN-e: 2663-1148

Periodicidad: Semestral

vol. 5 , núm. 1,2022

publicaciones.iniccperu@gmail.com

Recepción: 09 Septiembre 2021

Aprobación: 08 Octubre 2021

Publicación: 01 Enero 2022

URL: http://portal.amelica.org/ameli jatsRepo/390/3902822008/index.htm

Esta licencia permite a otros entremezclar, ajustar y construir a partir de su obra con fines no comerciales, y aunque en sus nuevas creaciones deban reconocerle su autoría y no puedan ser utilizadas de manera comercial, no tienen que estar bajo una licencia con los mismos términos.

\section{c) (7) (8)}

Esta obra está bajo una Licencia Creative Commons AtribuciónNoComercial 4.0 Internacional.
Resumen: El propósito de esta investigación fue analizar el marco normativo de la educación en el contexto global: internacional y nacional de Colombia. Se fundamentó teóricamente en los postulados de expertos en el área educativa, documentos y basamentos legales que permitieron contrastar diferentes concepciones sobre la temática en cuestión. El estudio se desarrolló bajo el paradigma cualitativo de corte exploratorio, desde la revisión documental. Entre los resultados más significantes se develó que los documentos generados en los eventos internacionales dan seguimiento y respuesta a la problemática educativa; asimismo, el marco normativo facilita la construcción de políticas públicas concebidas como justificación legal garantes del derecho a la educación para todos los ciudadanos, como un deber constitucional del cual depende la realización del proyecto de vida individual, colectivo y cultural en la sociedad, proporcionando así valor a los intangibles producto del conocimiento y del desarrollo sostenible.

Palabras clave: marco normativo, globalización, sociedad de conocimiento, políticas públicas, desarrollo sostenible.

Abstract: The purpose of this research was to analyze the normative framework of education in the global context: international and national of Colombia. It was theoretically based on the postulates of experts in the educational area, documents and legal bases that allowed contrasting different conceptions on the subject in question. The study was developed under the qualitative paradigm of an exploratory nature, from the documentary review. Among the most significant results, it was revealed that the documents generated in international events provide follow-up and response to educational problems; Likewise, the normative framework facilitates the construction of public policies conceived as a legal justification guaranteeing the right to education for all citizens, as a constitutional duty on which the realization of the individual, collective and cultural life project in society depends, thus providing value to intangibles product of knowledge and sustainable development.

Keywords: regulatory framework, globalization, knowledge society, public policies, sustainable development. 


\section{INTRODUCCIÓN}

La educación y por ende las universidades en la actualidad, han ampliado su función social, no sólo desplegando sus actividades en pro de educar, sino también por direccionar a sus actores hacia un desarrollo integral, que les permita un desenvolvimiento efectivo dentro de la sociedad cambiante en la que se encuentra inmersas, permitiendo la interrelación entre ellos; y en efecto, llegar en muchas oportunidades, a generar conocimiento basado en la experiencia y vinculado a las políticas públicas implementadas por los gobiernos nacionales.

Dentro de la simbiosis educativa mundial, es pertinente tener presente los roles que desempeñan las instituciones y sus actores para llevar adelante la gestión del conocimiento; no obstante, las instituciones educativas latinoamericanas dejaron de ser entidades cuya responsabilidad no era imperativa, y pasaron a ser permanentemente interpeladas por la colectividad, es decir, se le reclama el producto social de los recursos públicos invertidos en ella. Al respecto, Inciarte et al (2015) plantean que la universidad como institución responsable en la formación del talento humano, orienta su desarrollo a la generación de conocimiento dirigido a la solución de problemas contextuales, aunado a la construcción de capacidades coadyuvantes con el desarrollo humano y sustentable.

Por ello, es imperante para comprender la educación, analizar críticamente los aspectos intervinientes de este proceso, definiendo el marco legal que la contempla y su accionar como política pública. Según Pérez (2009) el marco legal compendia el conjunto de leyes, reglamentos, normas, decretos, entre otras publicaciones oficiales, donde se establece el basamento jurídico sobre el cual se sustenta cualquier protocolo investigativo.

Atendiendo a esas consideraciones, la educación como accionar principal en el desarrollo de los pueblos, requiere urgentemente su redimensión, exigida por el momento histórico que se vive, sin perder el norte establecidos por organismos internacionales que le dan sustento como fin principal en el desarrollo holístico del individuo. Al respecto, la Organización de las Naciones Unidas para la Educación, la ciencia y la Cultura [UNESCO] (2016), en el objetivo 4 del desarrollo sostenible plantea que la educación es la clave para poder alcanzar otros objetivos.

En el mismo orden de ideas, la Constitución Política de Colombia (1999), artículo 67, plantea que la educación es un derecho de la persona y un servicio público que tiene una función social, con ella se busca el acceso al conocimiento, a la ciencia, a la técnica, y a los demás bienes y valores de la cultura. De igual modo, la Constitución Nacional de Panamá (2004), artículo 91 expone el derecho que tienen los ciudadanos a la educación y la responsabilidad de educarse. En ese sentido, el Estado organiza y dirige el servicio público de la educación, garantizando a los padres de familia el derecho de participar en el proceso educativo de sus hijos; teniendo, acceso al conocimiento, la ciencia, la tecnología y a los valores culturales.

En relación con lo expuesto, se observa que en ambos países la educación es responsabilidad del Estado, instaurada como función social y derecho fundamental para todos los ciudadanos. Para los investigadores, las funciones de las instituciones educativas colombianas deben ajustarse al nuevo orden mundial, es decir, hacer reingeniería organizacional que permita responder a las necesidades de los individuos, en consonancia con los estándares legales. Urge, además, promover las funciones universitarias de docencia, extensión, investigación y gerencia, garantizando la instauración de un sistema multifuncional integrado por el Estado, universidades y empresas. Ante la problemática descrita, surge la siguiente pregunta de investigación: ¿De qué manera el marco normativo de la educación en el contexto internacional y nacional genera impacto en las políticas públicas educativas en Colombia?

La investigación se fundamentó en el paradigma cualitativo que según Hernández y Mendoza (2018) identifica la naturaleza profunda de la realidad estudiada, "su estructura dinámica, aquella que da razón plena de su comportamiento y manifestaciones, producto de técnicas para recabar información, como la revisión de documentos y su interpretación" (p.9). La misma, se enmarcó en un estudio exploratorio, pues se 
orienta hacia la identificación de los conceptos promisorios para indagar acerca de la temática seleccionada, preparando el terreno para estudios más amplios y profundos. (Muñoz, 2018)

Siguiendo con esa secuencia operativa y metodológica, se asumió la modalidad de revisión documental que desde la perspectiva de Ortiz (2015) se centra en el estudio de problemas con el propósito de ampliar y profundizar en el origen de éste, con apoyo de trabajos previos; así como, de información y datos divulgados por medios impresos o electrónicos. Para tal efecto, se empleó la técnica análisis de contenido, que para Álvarez Gayou (2016) representa el procedimiento más apropiado para el desarrollo de estudios cualitativos, que desde su alcance analiza mensajes, documentos, rasgos de personalidad entre otros aspectos subjetivos del ser humano como ente social. A partir de allí, se estableció la reflexión objeto de estudio, con el fin de analizar, recopilar, actualizar información sobre la problemática generando discusión, alcances y su aplicabilidad legal en la nueva sociedad del conocimiento.

\section{DESARROLLO}

\section{Abordaje teórico para la comprensión del problema}

En este abordaje, se analiza teóricamente el marco normativo de la educación a nivel internacional y nacional, caso Colombia; generando un proceso de comparación constante que permita develar el aporte legal, desde la perspectiva divergente de diferentes autores y normatividad jurídica vinculada a su aplicación en la política pública.

Considerando que la educación en la actual sociedad del conocimiento está signada por vertiginosos cambios producto de los efectos de la globalización, la pandemia y la nueva dinámica sociopolítica, se concibe desde la perspectiva legal, como una realidad compleja donde los agentes involucrados en el hacer educativo cuentan con herramientas y mecanismos disponible en la era digital, para garantizar su conectividad, se enfrenta a un nuevo reto: “...lograr una educación a escala global que abarque las necesidades de formación del capital humano con la finalidad de generar una praxis educativa integral, desarrollando desde y fuera del aula, habilidades, competencias y conocimientos de utilidad no solo en un entorno local, sino también mundial" (Sánchez Quintero, 2018, p.79).

De acuerdo con el informe de la Comisión Económica para América Latina y el Caribe (Cepal, 2002), el término globalización hace referencia al incremento de los procesos económicos, sociales y culturales de carácter mundial, llevando a la economía a globalizarse y generando nuevas políticas públicas que impactan en la educación.

Desde la perspectiva de los investigadores, esta situación debe adaptarse a los estándares legales exigidos por organismos internacionales y nacionales en busca de una educación de calidad que genere políticas públicas cónsonas con la nueva realidad exigida por la sociedad del conocimiento y los organismos responsables de darle seguimientos a esos procesos, ya que la educación, es el pilar fundamental para la construcción del conocimiento y del país. Esta reflexión lleva a considerar un análisis jurídico de la normatividad de la educación y su impacto a la sociedad del conocimiento en Colombia.

Análisis de la incidencia de la política educativa internacional en la política pública de Colombia

En este aparte se abordarán algunos documentos generados en conferencias mundiales, foros internacionales, tratados, pactos, convenios e instrumentos legales en orden jerárquico que le dan sustento a la praxis educativa en las instituciones de educación públicas a nivel internacional enfatizando en los casos: Panamá y Colombia. De acuerdo con la Organización de las Naciones Unidas para la Cultura, las Ciencias y la Educación [UNESCO] (1998) en la Conferencia Mundial sobre la Educación Superior, una educación superior en el siglo XXI y de conformidad con el párrafo 1, art. 26 de la Declaración Universal de Derechos Humanos, el acceso a la educación superior debe centrarse en los principios universales de igualdad e inclusión, sin discriminación de ninguna índole. En la declaración, se llegó a la conclusión en un compendio de acciones que deben prevalecer y aplicarse por el bien de la sociedad y la educación superior, 
donde la mayoría de los países apuesten al fomento de la investigación y la innovación con miras a impulsar su crecimiento económico sostenible, propiciar su desarrollo y conformar equipos multidisciplinarios con todos los actores responsables de las políticas nacionales e institucionales, garantizando así, la calidad y el compromiso del gobierno con el desarrollo educativo.

En el mismo orden de ideas, la UNESCO (2009) en la Conferencia mundial sobre la educación superior, planteó las siguientes líneas de acción: (a) La responsabilidad social de la educación superior recae en los gobiernos, apoyando los criterios de acceso, equidad y calidad, (c) Abarcar la cooperación internacional y redes entre instituciones en todos los ámbitos, (d) Financiación del aprendizaje, la investigación e innovación mediante iniciativas copartícipes entre el sector público y el privado. (e) Fomentar a través de la educación la gobernanza basada en responsabilidades, principios y alianzas estratégicas, $\mathrm{y}(\mathrm{f})$ Participar en el llamamiento a la acción y cooperación internacional para la formulación de estrategias sostenibles en educación superior e investigación a largo plazo.

Tratando de profundizar, la UNESCO (2016), plantea en el objetivo 4 del desarrollo sostenible que la educación es la clave para poder alcanzar otros objetivos de desarrollo sostenible (ODS). Es decir, cuando las personas pueden acceder a una educación de calidad, pueden ser excluidas del ciclo de la pobreza, contribuye a reducir las desigualdades y fomenta la igualdad de género, permitiendo empoderar a las personas con una vida más saludable y sostenible

$\mathrm{Al}$ respecto, la Conferencia Mundial sobre la Educación para el Desarrollo Sostenible (2015), pidió un compromiso renovado de todos los países, en la Declaración de Aichi-Nagoya que pongan en marcha el programa de acción mundial para la EDS y cumplir con las siguientes acciones: Promover políticas, integrar las prácticas de la sostenibilidad en los contextos pedagógicos y de capacitación, aumentar las capacidades de los educadores y formadores, dotar de autonomía a los jóvenes, instar a las comunidades locales y las autoridades municipales a que elaboren programas de EDS de base comunitaria.

En correspondencia con lo expuesto, en la Reunión mundial sobre la educación en Bruselas (2018) se destaca "la importancia del aprendizaje a lo largo de toda la vida" plasmada en su informe de cierre donde los mensajes de los participantes y compromisos asumidos acerca de cómo aprender en la vida, reafirmación el papel esencial de la educación, su formación, la enseñanza superior y la investigación como motores clave del desarrollo sostenible, abarcando el medio ambiente, el cambio climático y sus efectos, y no menos importante fortalecer la acción colectiva en relación con el ODS 4 en el marco de la agenda 2030.

Se requiere, además, dar seguimiento al compromiso de erradicar el analfabetismo mediante la educación formal y no formal, teniendo acceso equitativo a la capacitación digital en materia de medios de comunicación e información (TIC) como perspectiva de aprendizaje e implementación de las políticas y los planes pertinentes sobre la situación actual de la educación de adultos en todo el mundo.

Otro, documento analizado, fue el cuarto Informe Mundial sobre el Aprendizaje y la Educación de Adulto (2020), donde se concluyó que los progresos en la participación en el aprendizaje y la educación de adultos son generalmente insuficientes, los datos muestran desigualdades persistentes, profundas participación y que no está llegando a los principales grupos destinatarios, como los adultos con discapacidad, los adultos de edad avanzada, los grupos minoritarios y los adultos que viven en países en conflicto.

Por último, el Informe social mundial (2021) enfatiza en la necesidad de una transformación rural sostenible para alcanzar los ODS para 2030. Señala el acceso igualitario a la tierra, dándole participación e inclusión a la mujer; así como, garantizar el desarrollo cultural y económico de los pueblos indígenas con sus tierras ancestrales, proponer políticas de protección del agua, tierra y su restauración para ofrecer un futuro próspero.

Desde la óptica de los investigadores, estos documentos y acciones requieren del seguimiento de un equipo multidisciplinario integrados por todos los actores involucrados en la praxis educativa nacional e internacional y sus políticas sociales de acción, que garanticen un proceso inclusivo de calidad, socializador, tecnológico, de alianzas estratégicas que abarque la ciencia, la investigación y el trabajo de extensión con la 
finalidad de dar respuesta a las necesidades de la sociedad clave para conseguir los objetivos del desarrollo sostenible, como lo estipula la UNESCO (2016).

Marco normativo de la educación en Panamá

Para comprender el papel primordial que desempeña la educación en el en contexto mundial, con base en la similitud de este sistema en cuanto al desarrollo de su praxis todo lo que engloba su accionar, se recurre a la Constitución Política de la República de Panamá (2004) en su capítulo $5^{\circ}$ sobre la educación, la conciben como un derecho fundamental, consagrado en el Art. 91 que expresa: "Todos tienen el derecho a la educación y la responsabilidad de educarse".

Desde esa óptica jurídica, la carta política panameña consagra a la educación como derecho y servicio público, cuya responsabilidad recae directamente en el Estado, conforme a las exigencias de la sociedad y la familia, como garantía de la calidad del servicio. En efecto, promulgan su gratuidad a nivel oficial, sin causar menoscabo a aquellas instituciones de carácter privado. Es pertinente, además, referir los artículos 94,95,96,99 de esta ley macro, vinculados a la libertad de enseñanza, gratuidad de la educación oficial en todos los niveles pre universitarios, responsabilidad del Estado en la organización, supervisión y planeación de programas, planes de acuerdo con las necesidades nacionales y el reconocimiento de los títulos académicos y profesionales expedidos por los agentes competentes autorizados por el Estado. Es importante destacar en este punto, que la universidad oficial del Estado fiscalizará a las universidades particulares aprobadas oficialmente para garantizar los títulos que expidan y revalidará los de universidades extranjeras en los casos que la Ley establezca.

Se analizó así mismo en el contexto panameño, la Ley Orgánica de Educación (2003) donde se establecen las disposiciones fundamentales relacionadas a los principios, fines y normas de la educación, reconociendo el derecho que tienen los niños y jóvenes residentes en el país, de recibir una educación de calidad. La misma se divide en oficial y particular; el sistema educativo está compuesto por dos subsistemas (regular y no regular); en él se establece que la educación permanente es obligatoria; además se exponen los fines de la educación panameña. La misma se caracteriza como democrática y el ministerio de educación es el encargado de autorizar y fijar los planes de estudios, supervisar, organizar los programas de enseñanza y la organización de las escuelas pre-primaria, primaria y secundaria oficial del país.

En cuanto al nivel de educación superior, el Ministerio de educación (1999) promulga el Decreto 50 de (1999) "Por el cual se regula el funcionamiento de los centros de enseñanza superior, oficiales y particulares, y se dictan otras disposiciones.” En el mismo orden, la Ley 34 de 6 de julio de 1995, Art. 60, establece que el tercer nivel de enseñanza o educación superior será impartido en las universidades, en centros de enseñanza superior y en centros de educación postmedia, revistiendo especial importancia para el desarrollo del país.

En la actualidad, la estrategia decenal de modernización de la educación panameña establece en la ley antes mencionada, nuevos criterios que propician e impulsan el funcionamiento, desarrollo y evaluación de esta modalidad educativa; apoyado los Art. 1 y 2 , referidos a que cuya la finalidad de los centros de enseñanza superior es la formación de profesionales en los distintos campos de la investigación y de la actividad humana, la extensión científica, técnica y cultural; así como, servicios profesionales y de asesoría para la satisfacción de las necesidades de los egresados del segundo nivel de enseñanza o educación media y de la demanda de recursos humanos y sus respectivos objetivos.

Desde la óptica de los investigadores, estos documentos e instrumentos legales deben ser implementados, supervisados y transformado en políticas públicas, aplicados por los entes gubernamentales responsables de la educación y permitan la vinculación con las instituciones de educación superior como rectores de la praxis educativa y la formación de talento humano. Se requiere, además, realizar procesos de reingeniería organizacional dando respuesta a la demanda de la sociedad actual para así, accionar mecanismos de financiación en investigación ya que es clave para conseguir los objetivos del desarrollo sostenible, según los lineamientos de la UNESCO.

Análisis de la incidencia de la política educativa nacional en la política pública colombiana 
La educación colombiana está regida por la Constitución Política de Colombia(1991), Art. 67 se entiende a la educación como un derecho fundamental y servicio público gratuito cuya prestación es responsabilidad del Estado. conforme a las exigencias de la sociedad y la familia. Esta es obligatoria desde los 5 años hasta los 15 años, abarcando la enseñanza preescolar, básica primaria y secundaria; será prestada por instituciones educativas del estado, sin causar menoscabo a aquellas instituciones de carácter privado.

En el mismo orden de ideas, expresa el citado instrumento legal, que la educación formará al colombiano en el respeto a los derechos humanos, a la paz, a la democracia; a la práctica del trabajo y la recreación, para el mejoramiento cultural, científico, tecnológico y para la protección del ambiente. Asimismo, el estado estará vigilará, organizará, planificará y supervisará el hecho educativo, garantizando la ampliación de su cobertura, la permanencia de los educandos y la calidad del servicio. Además, la nación y las entidades territoriales participarán en la dirección, financiación y administración de los servicios educativos estatales, en los términos que señalen la constitución y la ley.

Otro instrumento importante en esta revisión es la Ley 115 (1994), Art. 1 que plantea el objeto de la educación, concebida como un proceso de formación permanente, personal, cultural y social, que se fundamenta en una concepción integral de la persona humana, de su dignidad, derechos y deberes. Además, esta ley señala las normas generales para regular el servicio público de la educación que cumple una función social acorde con las necesidades e intereses de las personas, de la familia y de la sociedad. Es relevante resaltar en esta ley sus fines, aunados al respeto a la vida, los derechos humanos, la creación y fomento de una conciencia de la soberanía nacional para la práctica de la solidaridad y la integración con el mundo, en especial con Latinoamérica y el Caribe. También, promulga, el acceso al conocimiento, la ciencia, la técnica y valores de la cultura, el fomento de la investigación y el estímulo a la creación artística en sus diferentes manifestaciones.

La situación antes descrita, se concreta en Ley 30 (1992), Art. 35 referente a la educación media y su articulación con la educación superior. Esta es un proceso permanente que posibilita el desarrollo de las potencialidades del ser humano de una manera integral, se cursa después de la educación media y tiene por objeto el pleno desarrollo de los alumnos y su formación académica o profesional. Además, es un servicio público cultural, inherente a la finalidad social del estado quién de conformidad con la Constitución Política de Colombia y la presente Ley, garantiza la autonomía universitaria cumpliendo funciones de velar por la calidad del servicio educativo a través de la supervisión y vigilancia, generando libertad de pensamiento, pluralidad ideológica en un marco de libertades de enseñanza, de aprendizaje, de investigación y de cátedra.

Establece la citada ley, Art. 6 la necesidad de profundizar en la formación integral de los colombianos dentro de las modalidades y calidades de la educación superior, capacitándolos para cumplir las funciones profesionales, investigativas y de servicio social que requiere el país como factor de desarrollo científico, cultural, económico, político y ético a nivel nacional y regional. De igual modo, expone que urge promover la unidad nacional, la descentralización, la integración regional y la cooperación interinstitucional con miras a que las diversas zonas del país dispongan de los recursos humanos y de las tecnologías apropiadas que le permitan atender adecuadamente sus necesidades, en consonancia con las comunidades académicas y la articulación con sus homólogas a nivel internacionales. Este proceso, debe ser consonó con los campos de acción, requerimientos y programas académicos.

Análisis de la incidencia de la política educativa departamental en la política pública de Colombia

El servicio educativo departamental está regulado por la ley 115 (1994) la cual responde a los presupuestos planteados por la Constitución Política, tales como, responsabilidad del Estado, la sociedad y la familia en la calidad del servicio educativo, la prestación del servicio, a través de entidades territoriales certificadas. En correspondencia, el Decreto 1860 (1994), establece que todas las personas que residan en territorio colombiano recibirán educación prescolar y básica, en instituciones educativas de carácter público o privado.

La financiación de la educación pública colombiana, según Ley 115 (1994), Art. 175, se realizará con recursos provenientes "el situado fiscal, con los demás recursos públicos nacionales dispuestos en la ley, más el 
aporte de los departamentos, los distritos y los municipios", de acuerdo con lo dispuesto en la Ley 60 (1993) derogada por la ley 715 (2001), la cual regula la prestación del servicio educativo.

En el mismo orden, la Constitución Política de Colombia (1991), Art. 286 refiere que las entidades territoriales, son los departamentos, distritos, municipios y territorios indígenas. Para los efectos de financiación del servicio educativo, la ley 715 (2001), Art. 20, define las entidades territoriales certificadas como aquellas "certificadas según la ley, los departamentos y los distritos". En efecto, la nación certificará a los municipios con la cantidad de habitantes, a través del DANE para efectos del cálculo poblacional. Según el censo, los recursos se asignan de acuerdo con la prestación efectiva del servicio.

La distribución de los recursos para educación es realizada por el Departamento Nacional de Planeación, a quien se le confirió esa competencia, en virtud de lo consagrado en la ley 1753 (2015), Art. 165 sobre la competencia, que anteriormente ejercía el Consejo Nacional de Política Económica y Social (CONPES), entidad encargada de autorizar la asignación de los recursos. Estos criterios utilizados para la asignación de los recursos del sistema general de participación para educación se encuentran en la Ley 715 (2001), Art. 16 donde se manifiesta que la población atendida, se aplica de acuerdo con los estudiantes matriculados oficialmente al año anterior.

Análisis de la incidencia de la política educativa institucional en la política pública de Colombia

A nivel institucional la educación en Colombia se rige por la Constitución Política de Colombia (1991), por la Ley General de Educación (1994), las directrices emitidas por el Ministerio de educación y su Plan de desarrollo de gobierno. Además, el servicio educativo departamental es regulado por la ley 115 de 1994 en su artículo 175 referido al pago de salarios y prestaciones de la educación estatal.

La distribución de los recursos para educación es realizada por el Departamento Nacional de Planeación, a quien se le confirió esa competencia, en virtud de lo consagrado la Ley 1753 (2015), Art. 165 otorgándosele autonomía administrativa a las instituciones educativas sin precedentes. Lo expuesto, permitió la descentralización que incidió directamente en acciones institucionales como gestión de recursos, proyección de currículos y diseño del Proyecto educativo institucional (PEI) siendo éste, la estrategia fundamental ordenada por la Ley 115 de 1994, para propiciar la transformación de las instituciones como ejes de desarrollo y mejoramiento de la calidad educativa humana e institucional, ya que es una construcción colectiva, que conlleva al crecimiento, desarrollo escolar y social de las comunidades educativas.

De igual forma, Decreto 1860 (1994), Art. 16 plantea la obligatoriedad del proyecto educativo institucional para las instituciones educativas oficiales y privadas. Además, deben presentar evidencias de los ajustes y avances (informe) a la secretaría de educación y de no cumplir serán sancionadas como lo estipula la ley. Al respecto, González \& Duque (2008) expresan la necesidad imperiosa de diseñar políticas exitosas con el objetivo de mejorar las condiciones de vida de la población, implantar nuevas formas de relación entre sociedad y estado, mejorar el desempeño de las instituciones; así como, reflejar transparencia y responsabilidad en las acciones del gobierno para reforzar la confianza de la sociedad y aproximarse a las políticas públicas en la ruta de la creación de una sociedad sustentable y democrática.

\section{CONCLUSIONES}

$\mathrm{Al}$ analizar el marco normativo y los documentos generados en los eventos de la educación en el contexto internacional y nacional de Colombia se develó: crecimiento y progreso en el ámbito educativo, disociado de una política nacional en relación con lo investigado y la designación de líneas estratégicas que permitieron un comportamiento en red, esto aparejado con la falta de continuidad político-estratégica en materia de explotación científico-tecnológica. Como evidencia tangible, se tiene el subsidio otorgado a múltiples investigaciones con poca pertinencia y relevancia nacional, redundando en la fuga de talentos hacia espacios que fuesen más congruentes con la formación recibida. 
De igual modo, se comprobó la necesidad de generar acciones direccionadas hacía la construcción de políticas públicas con filosofía democrática, teniendo en cuenta una visión articulada de los problemas, crear mecanismos de control social, buscar calidad, universalidad y generar redes para su proceso de masificación, evaluación y supervisión.

Así, se concibe a las políticas públicas como acciones mancomunadas gubernamentales y sociales, en busca de un objetivo específico. En efecto, se deben gestionar políticas públicas educativas desde donde se garantice, el derecho a la educación para todos los ciudadanos, como servicio público y derecho constitucional que permita el desarrollo integral en lo individual, colectivo, cultural en la sociedad, acorde a los estándares, acciones y lineamientos generados de los tratados internacionales y la legislación del país.

En síntesis, los entes gubernamentales deben proponer políticas educativas encaminadas a disminuir el marco índice de desigualdad, dando oportunidad a todos los ciudadanos a formarse. Para ello se debe invertir capacitar el talento humano y en la alfabetización tecnológica, con miras a desarrollar una práctica de avance sociocultural, enmarcado en las oportunidades que ofrece la era digital en la actualidad. Además, se requiere la inclusión tecnológica a toda la ciudadanía, sin discriminación; invertir en investigación, ciencia y tecnología; generar redes nacionales e internacionales, conformar equipos multidisciplinarios cualificados en educación y darles seguimientos a las acciones producto de eventos académicos y propuestas hechas por organismos internacionales para la integración a la sociedad del conocimiento y el desarrollo sostenible.

\section{ReFERENCIAS}

Álvarez Gayou, J. (2016). Cómo hacer investigación cualitativa. Fundamentos y metodologías. Paidós Educador.

Cepal (2002). Globalización y Desarrollo. ONU. https://repositorio.cepal.org/bitstream/handle/11362/2724/2/ S2002024_es.pdf

Congreso de la República de Colombia. (1994, 8 de febrero). Ley 115. Por la cual se expide la Ley General de Educación. Diario Oficial 41214. https://bit.ly/3237N87

Constitución Política de Colombia. (1991). Gaceta Constitucional n. ${ }^{\circ} 116$. http://bit.ly/2NA2BRg

Constitución Política de la República de Panamá. (1994). Gaceta oficial n. ${ }^{\circ 215176 . ~ h t t p s: / / w w w . i l o . o r g / d y n / t r a v a i l / ~}$ docs $/ 2083 /$

Decreto 1860 de 1994. Por el cual se reglamenta la ley 115 de 1994 en los aspectos pedagógicos y organizativos generales. 3 de agosto de 1994. Diario Oficial 41480.

González, E. \& Duque, S. (2008). Sobre el marco normativo para formular una propuesta de Políticas públicas para la formación por ciclos y la evaluación por competencias en la educación superior colombiana. Revista Opinión Jurídica, 7(14), 83-98. http://www.redalyc.org/articulo.oa?id=94512646006

Hernández, R., \& Mendoza, C. (2018). Metodología de la investigación. Las rutas cuantitativa, cualitativa y mixta. Editorial McGrawHill Education.

Inciarte, A, Parra, M., \& Bozo, A. (2015). Reconceptualización de la universidad. Una mirada desde América Latina. Editorial Astro Data, S.A.

Ley 115 de1994. Por lo cual se expide la ley general de educación. 8 de febrero de 1994. Diario Oficial n. 41214.

Ley 34 de 6 de julio de (1995). Educación superior. Ministerio de educación de Panamá. (1999). Decreto 50 sobre educación superior.

Ley 47 de 1946. Ley Orgánica de Educación de Panamá. 24 de septiembre de 1946.

Ortiz, A. (2015). Enfoques y métodos de investigación en las ciencias sociales y humanas. Ediciones de la U. Bogotá.

Pérez, A. (2009). Guía metodológica para anteproyectos de investigación (3ra ed.). Edición: FEDUPEL.

Sánchez Quintero, J. (2018). Educación y administración en un mundo globalizado. Editorial Unimagdalena.

Unesco. (2009). Conferencia Mundial sobre la Educación Superior. La nueva dinámica de la educación superior y la investigación para el cambio social y el desarrollo. https://t.co/Zw3rI7IEob. 
Manuel Facundo Villero Pacheco, et al. Análisis del marco normativo de la educación en el contexto...

Unesco. (2014a). Conferencia Mundial sobre la Educación Superior. La educación superior en el siglo XXI: Visión y acción. https://www.iesalc.unesco.org/ess/index.php/ess3/article/view/171.

Unesco. (2014b). Conferencia Mundial sobre la Educación para el Desarrollo Sostenible. http://www.unesco.org/ new/es/unesco-world-conference-on-esd-2014/.

Unesco. (2016). Desglosar el Objetivo de Desarrollo Sostenible 4. Educación 2030. Guía. Unesco. https:// es.unesco.org/sdgs.

Unesco. (2018). Reunión Mundial sobre la Educación 2018. Declaración de Bruselas. https://cerlalc.org/ publicaciones/reunion-mundial-sobre-la-educacion-2018-declaracion-bruselas/.

Unesco. (2019). El Informe Mundial sobre el Aprendizaje y la Educación de Adultos GRALE 4. https:// uil.unesco.org/es/educacion-adultos/informe-mundial-sobre-el\%20aprendizaje-y-la-educacion-de-adultos.

United Nations. World Social Report (2021). Reconsidering rural Development. Informe Social Mundial de UNDESA 2021. https://www.un.org/development/desa/dspd/world-social-report/2021-2.html. 